\title{
Modelling the Influence of Heterogeneous Annulus Material Property Distribution on Intervertebral Disk Mechanics
}

Journal Article

Author(s):

Marini, Giacomo; Ferguson, Stephen J. (1)

Publication date:

2014-08

Permanent link:

https://doi.org/10.3929/ethz-b-000087528

Rights / license:

In Copyright - Non-Commercial Use Permitted

Originally published in:

Annals of Biomedical Engineering 42(8), https://doi.org/10.1007/s10439-014-1025-5 


\title{
Modelling the Influence of Heterogeneous Annulus Material Property Distribution on Intervertebral Disk Mechanics
}

\author{
Giacomo Marini and Stephen J. Ferguson \\ Institute for Biomechanics, ETH Zurich, HPP-O14, Hönggerbergring 64, 8093 Zurich, Switzerland \\ (Received 8 January 2014; accepted 2 May 2014; published online 14 May 2014)
}

Associate Editor Scott I Simon oversaw the review of this article.

\begin{abstract}
Accurate modeling of the annulus fibrosus (AF) is a crucial aspect to study spine mechanics in silico. Numerical models require validation at both the microscale and organ level to be representative of the real system. Although the AF presents distributed material properties, its response is often modeled with a homogeneous stiffness distribution. The aim of this study was to investigate the influence of different modeling approaches on the numerical response of disk models, based on lamellae mechanics. A material mapping strategy was developed to define element-wise the local annulus material properties, based on prior findings of singlelamella mechanics and collagen distribution. Three modeling approaches were compared: homogeneous, radial and radialcircumferential distribution of material properties. The simulations showed a strong influence of the chosen modeling approach on the disk's tissue- and organ-scale mechanics. A homogeneous model with uniform, average lamellae stiffness predicted a substantially different internal stress distribution and organ-level response, compared to a model with heterogeneous material properties of the annulus lamellae. Finally, the study has indirectly highlighted that the organization of the mature disks could be a consequence of adaptation to the stresses induced by the applied loads, in order to evenly distribute the load over the entire structure.
\end{abstract}

Keywords-Annulus modeling, Lamellae stiffness, Collagen fibers.

\section{INTRODUCTION}

The mechanical response of physiological structures depends on both the biochemical composition and structural organization of their constituents. In general, the mechanical response of soft tissues is related to the collagen type and distribution. ${ }^{18}$ Depending on the tissue, the collagen fibers can provide a network

Address correspondence to Giacomo Marini, Institute for Biomechanics, ETH Zurich, HPP-O14, Hönggerbergring 64, 8093 Zurich, Switzerland. Electronic mail: gmarini@ethz.ch supporting other macro-molecules (e.g., hyaline cartilage) or have a direct load-bearing function (e.g., tendons).

The intervertebral disk (IVD) owes its complex mechanical response to the interaction of two adjacent soft tissues, in which the collagen fibers have different structural tasks. The disk anatomy comprises a central gelatinous core, the nucleus pulposus (NP), which is surrounded by alternating, fiber-reinforced lamellae (from 15 to 25 distinct layers), the AF. ${ }^{10}$ In the NP, the collagen fibrils, mostly collagen type II, are organized as a randomly dispersed network, which provides support for glycosaminoglycans (GAGs) and other molecules. The annulus lamellae are composed of collagen fibers, mostly type I and some type II, embedded in a biological ground matrix. ${ }^{32}$ Within each lamella, the collagen fibers are aligned along preferential directions which vary depending on the location within the $\mathrm{AF}^{8,32}$

Several studies have shown that the IVD, and in particular the AF, demonstrates a heterogeneous spatial distribution of its material properties. $2,11,26,32,43,44$ In particular, the anterior outer region of the AF has been shown to be stiffer than the posterior outer region. Furthermore, the outer regions of the AF are stiffer than the inner region, closer to the $\mathrm{NP}^{26}$ (Fig. 1a). Although numerical models have demonstrated that the local fiber orientation may partially explain the regional differences of stiffness, ${ }^{13,31}$ an approach focused only on fiber orientation fails to explain and incorporate the variable response of single lamellae when stretched along the fiber direction. ${ }^{26}$ In general, this could be explained by the heterogeneous distribution of the collagen content itself, which has been found to vary radially. ${ }^{7}$ However, no significant correlation has been found between the total collagen content and mechanical parameters i.e., tensile modulus, strength of single lamellae. ${ }^{43}$ 
(a)

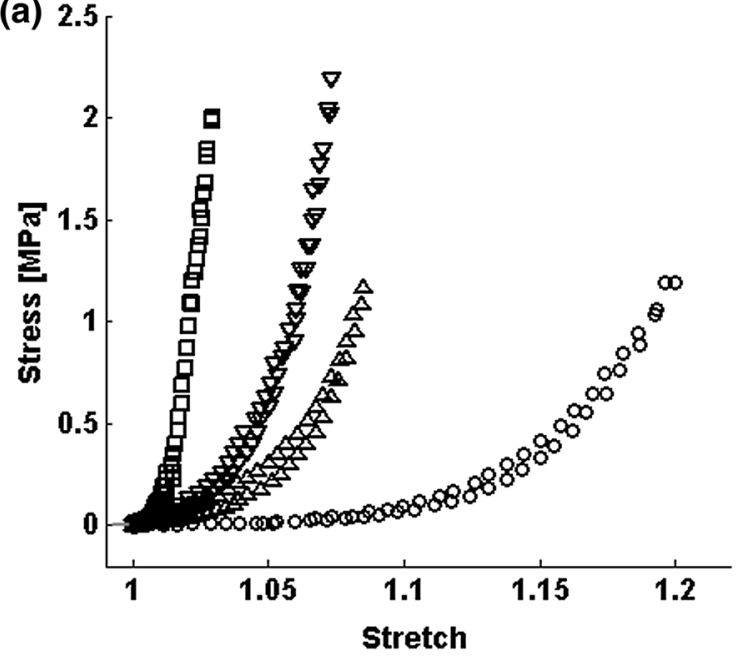

a ant. outer (b) 2.5

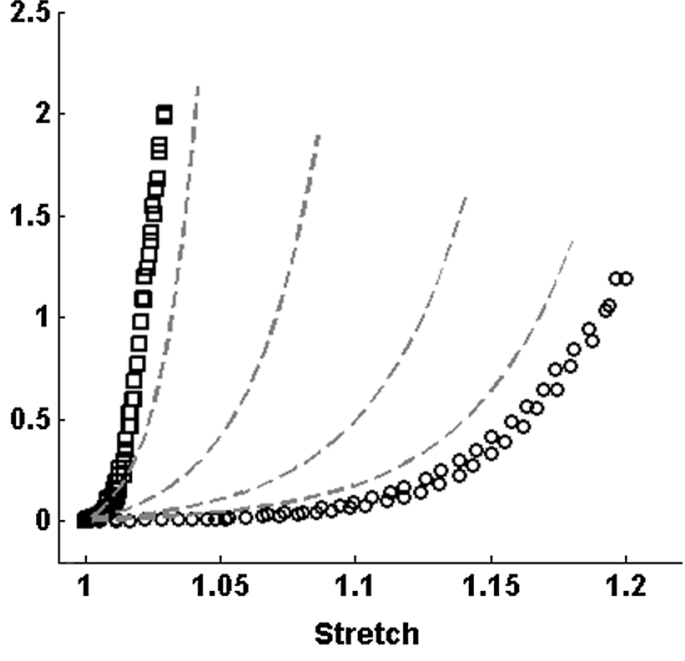

- post. inner $\quad---\cdot$ mid lamellae

FIGURE 1. (a) Typical stress-stretch curves of lamellae from four regions of the $\mathrm{AF}^{26}$ (b) Stress-stretch curves for the radial distribution model (RDM) computed by the proposed algorithm (dashed gray lines) based on the collagen type I distribution for 1318 years subjects. The AF was divided into four stiffness regions.

Nevertheless, it is plausible that the individual collagen type distribution, rather than the total collagen content, should be considered to fully assess the local lamellae stiffness. Eyre and Muir showed that the proportions of collagen type I ([Coll $]$ ) and type II ([Coll $\mathrm{II}])$ vary gradually and inversely across the AF, with type I content decreasing from the outer to the inner annulus. ${ }^{14}$ Brickley-Parsons and Glimcher ${ }^{7}$ further investigated the chemistry of collagen and reducible collagen crosslinks, dividing the disk cross-section in four areas: anterior, posterior and two lateral regions. These authors reported that no significant changes in collagen content and type were detected circumferentially around the same disk and between different spinal levels for the group of specimens in the 13- to 18-year-old age group. The changes were mostly in the radial direction, in agreement with Eyre and Muir. However, circumferential changes were seen in samples from older donors, 36-82 years of age, where both collagen type (Fig. 2a) and collagen crosslink density varied. In experimental studies, where the lamellae mechanics were investigated, specimens were tested from donors mostly in the second age group (Table 1). However, Galante ${ }^{17}$ reported that the elongation, residual deformation and energy dissipation decreased progressively until age 26, after which they remained practically constant. Skrzypiec et al. ${ }^{44}$ reported that the ultimate tensile strength of the outer annulus for male disks decreased by $66 \%$ over the age range $48-96$ years.

The research community now makes extensive use of numerical simulations to investigate the IVD's response to different loads. ${ }^{27,48}$ However, those studies mostly model the AF by assuming a homogeneous stiffness distribution, which is not the case for the physiological disk. The fidelity of the material model is even more relevant when using an inverse approach to derive the local material properties of the annulus based on experimental force-displacement data at the organ level. Some studies model heterogeneous material properties of the AF by representing the collagen fibers as discrete bar elements ${ }^{41,42}$ or through rebar brick elements. ${ }^{38}$ In these, a radial distribution of the stress-strain relationship for the collagen was assumed, adjusted to the relative collagen content of collagen type $\mathrm{I}_{\text {and }} \mathrm{II}^{7}$ in the AF. However, neither the full heterogeneity of annulus properties nor the actual uniaxial tensile response of individual lamellae has been explicitly implemented in organ-scale models.

Therefore, assuming that the AF lamellae play a critical role in the disk's mechanical response, especially if one considers aging, adaptation or degeneration, the purpose of the present study was to investigate the influence of different modeling approaches on the numerical response of disk models, based on lamellae mechanics. Specifically, a material mapping strategy was developed to define the local annulus material properties on a per-element basis. Two different scenarios were investigated: a heterogeneous radial distribution of the lamellae stiffness (Radial Distribution Model, RDM), and a heterogeneous radial-circumferential distribution of the lamellae stiffness (Radial Circumferential Distribution Model, RCDM), based on the previously reported heterogenous - and age-dependent - distribution of collagen type and content. ${ }^{7}$ A model with a homogeneous distribution of the lamellae stiffness (Homogeneous 

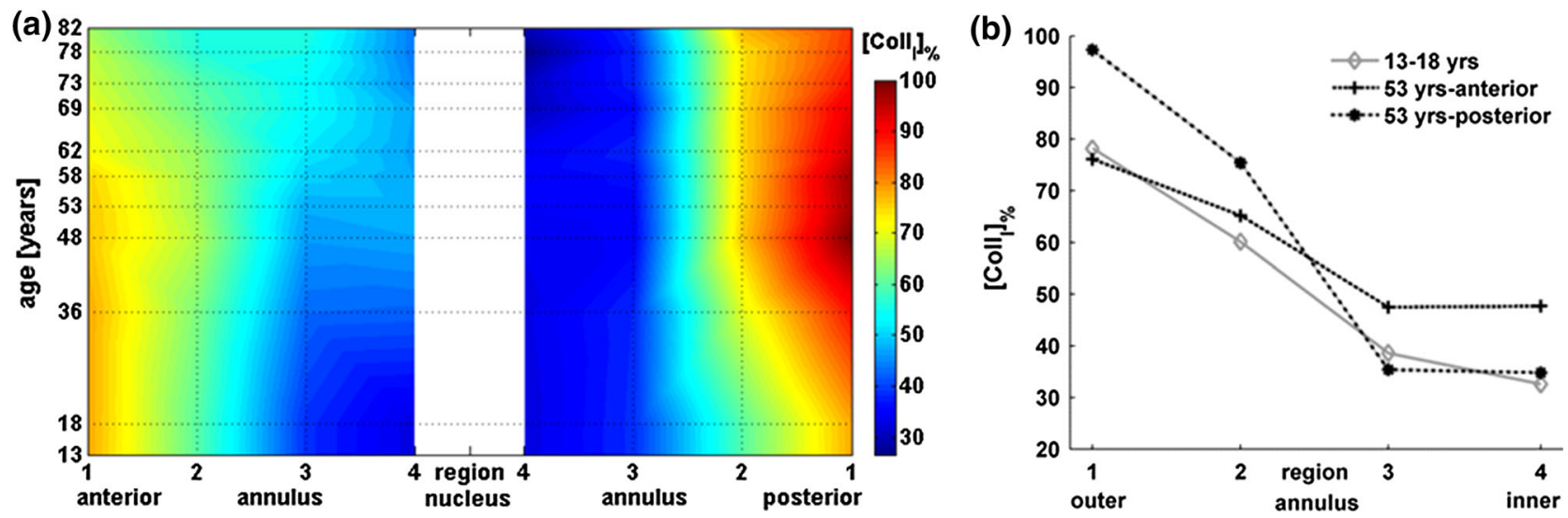

FIGURE 2. (a) Relative proportion of type I collagen in the lamellae of the AF vs. age for an L2-L3 disk. Color map expressed as \% dry weight of total collagen content. (b) Distribution of type I collagen vs. annulus region for 13-18 years group (gray), and 53 years (black). Data adapted from Brickley-Parsons and Glimcher. ${ }^{7}$

TABLE 1. Studies of lamellae mechanics.

\begin{tabular}{|c|c|c|c|}
\hline Study & Specimen & Age (years) & Level \\
\hline Acaroglu et al. ${ }^{1}$ & Multiple-lamellae & $2-88$ & L3-L4 \\
\hline Ebara et al. ${ }^{12}$ & Multiple-lamellae & $26-53$ & L3-L4 \\
\hline Galante $^{17}$ & Multiple-lamellae & $5-78$ & Not reported \\
\hline Holzapfel et al. ${ }^{26}$ & Single lamellae & $57.9($ mean $) \pm 15.4(\mathrm{SD})$ & L2-L3 \\
\hline Skaggs et al. ${ }^{43}$ & Single lamellae & Not reported & L1-L2 \\
\hline Skrzypiec et al. ${ }^{44}$ & Multiple-lamellae & $48-91$ & T8-S1 \\
\hline
\end{tabular}

Distribution Model, HDM) was used as a reference for the standard modeling approach in disk mechanics. Given the different stiffness distribution, we expect that each model will predict a substantially different response at both the micro (stress patterns) and organ scale. For each study case, we investigated the response of the disk to four different quasi-static load cases.

\section{MATERIALS AND METHODS}

\section{Model}

A representative L2L3 disk was defined with a major axis of $50 \mathrm{~mm}$ and a minor axis of $37 \mathrm{~mm}$. A height of $9.5 \mathrm{~mm}$ was defined. The volume of the NP was set to $42 \%$ of the entire disk geometry. ${ }^{49}$ The disk bulge was assumed to vary linearly from $2.5 \mathrm{~mm}$ on the inner AF to $1 \mathrm{~mm}$ on the outer AF. The AF was assumed to be comprised of ten alternating layers, with the fiber ( $\mathrm{fb}$ ) orientation described as in Holzapfel et $a l .{ }^{26}$ Inclined rigid bony endplates were added at the top and bottom $\left(2^{\circ}\right.$ with respect to the transversal disk section). The inferior endplate was rigidly fixed in the three translational degrees of freedom to approximate the usual experimental constraints of an isolated motion segment with the vertebrae embedded cranially and caudally. ${ }^{48}$ The loads were applied by means of displacement or rotation of the superior endplate. Linear fully-integrated hexahedral elements were used for the discretization of the system. The average seeding length was defined by the ratio of the disk height over the number of layers of the AF.

\section{Material Properties}

The response of the IVD tissues is highly nonlinear. Therefore, a large deformation approach was used to describe the mechanical response of the AF and NP tissue. The constitutive equations were derived by assuming a specific strain energy function $\Psi_{i}$ with $i=\{\mathrm{np}, \mathrm{af}, \mathrm{fb}\}$ for each tissue. A Neo-Hookean model was used for the NP, assuming a homogeneous, incompressible material, Eq. (1). The annulus was represented as a composite material of collageneous fibers embedded in a ground matrix. The fibers were not included as discrete elements, but described in a continuum manner, incorporating their initial orientation. Each component was described by a different hyperelastic formulation. A modified Yeoh's model has been used for the ground matrix of the AF, assuming a homogeneous, incompressible material, Eq. (2).

$$
\Psi(C)_{\mathrm{np}}=C_{10}^{\mathrm{np}}\left(\bar{I}_{1}-3\right)+\frac{\kappa^{\mathrm{np}}}{2}(J-1)^{2}
$$




$$
\Psi(C)_{\mathrm{af}}=C_{10}^{\mathrm{af}}\left(\bar{I}_{1}-3\right)+C_{20}^{\mathrm{af}}\left(\bar{I}_{1}-3\right)^{2}+\frac{\kappa^{\mathrm{af}}}{2}(J-1)^{2},
$$

where $J=\operatorname{det}(\mathbf{F})$ is the Jacobian determinant, measure of the volume change. $\bar{I}_{1}$ is the first invariant of the deviatoric deformation tensor $\overline{\mathbf{C}}=J^{-2 / 3} \mathbf{C}, \mathbf{C}=\mathbf{F}^{\mathrm{T}} \mathbf{F}$ is the right Cauchy-Green deformation tensor, $\mathbf{F}$ is the deformation gradient tensor, a measure of the transformation, $C_{10}^{\mathrm{np}}, \kappa^{\mathrm{np}}, C_{10}^{\mathrm{af}}, C_{20}^{\mathrm{np}}$ and $\kappa^{\mathrm{af}}$ are the material coefficients, with unique values for nucleus and annulus tissue. These parameters have been defined by the analysis of experimental data from literature (see Table 2).

\section{Collagen Fibers Response}

Assuming that the stiffness of the lamellae is related to the local $\left[\mathrm{Coll}_{\mathrm{I}}\right]$, the heterogeneous mechanical properties of the annulus were described by varying the lamellae stiffness to reflect the local [Coll $]$ ], combining and relating observations from the papers of Holzapfel et $a l^{26}$ and Brickley-Parson and Glimcher. ${ }^{7}$ According to the uniaxial tensile tests performed by Holzapfel et al. $^{26}{ }^{26}$ (Fig. 1a) the stress-stretch response of the lamellae of the annulus boundaries were used as reference curves. To compute the intermediate lamellae response (from the inner to the outer annulus, Fig. 1b), the curves were scaled radially, based on the local [Coll $\mathrm{I}]$ from Brickley-Parson and Glimcher. ${ }^{7}$ Then, the individual curves were parameterised by fitting a polynomial hyperelastic constitutive equation to determine the local material parameters Eq. (3). Their response was modeled as tension only.

$$
\Psi(C)_{\mathrm{fb}}=\frac{\mu_{i}}{\gamma_{i}}\left(I_{4}^{\gamma / 2}-1\right)-\mu_{i} \ln \left(I_{4}^{1 / 2}\right) \text { if } I_{4} \geq 1,
$$

0 otherwise,

where $I_{4}$ is the square of the fiber stretch $I_{4}=$ (F fiber) $\cdot(\mathbf{F}$ fiber) where fiber is the fiber direction in the reference configuration. $\mu_{i}$ and $\gamma_{i}$ are coefficients describing the local lamellae stiffness. These parameters were assigned to each element of the AF together with the local fiber direction.
For the RCDM, the lamellae responses were related to the distribution of the 53 year old case from Brickley-Parson and Glimcher (Fig. 2b), since the average age of the specimens tested by Holzapfel et $a l .{ }^{26}$ was 57.9 years. The parameters of the circumferentially intermediate lamellae were assigned by linear interpolation with respect to the angular position of the element around the annulus. For the RDM, the distribution of $\left[\mathrm{Coll}_{\mathrm{I}}\right]$ of the 13-18 year old group was used, as $\left[\mathrm{Coll}_{\mathrm{I}}\right]$ was seen to vary only radially in this group. This distribution was related to the lamellae response of the anterior-outer lamellae (square mark, Fig. 1a) and of the posterior-inner-lamellae (circle mark, Fig. 1a). These curves were selected because of the small change of [Coll ${ }_{\mathrm{I}}$ ] until the age of 53 years in these regions of the $\mathrm{AF}(\% \approx 4)$. The above procedure was incorporated within an algorithm which computes and implements the local fiber direction. ${ }^{35}$ In Fig. 3, the value of $\gamma_{i}$ are plotted over the IVD mid crosssection for the RDM and RCDM. Three HDMs were used to evaluate the disk response with homogeneous properties: (i) HDM-AV, a model with lamellae response equal to the average lamellae stiffness of the inner and outer lamellae of the RDM (the response was computed by assuming the average $\left[\mathrm{Coll}_{\mathrm{I}}\right]$ the anterior-outer-lamellae and the posterior-inner-lamellae); (ii) HDM-I, a model with lamellae response equal to the inner lamellae response of the RDM; (iii) HDM$\mathrm{O}$ a model with lamellae response equal to the outer lamellae response of the RDM.

\section{Numerical Simulations}

The constitutive equations have been implemented in the ABAQUS finite element package (ABAQUS 6.12.3, Hibbit, Karlsson and Sorenson Inc., Pawtucket, RI, USA) with user-defined Fortran subroutines. No time dependent effect was considered.

Assuming the swelling of the AF ground matrix is negligible compared to the NP swelling, ${ }^{19,28,38}$ the effect of the internal hydrostatic pressure on the stress distribution in the AF was considered by performing a pre-stress analysis. This was conducted as the first step of the analysis and used an algorithm specially devel-

TABLE 2. Finite element model material properties of the ground matrix.

\begin{tabular}{lcccc}
\hline Nucleus ground matrix & & \multicolumn{2}{c}{ Annulus ground matrix } \\
$C_{10}^{\mathrm{np}}(\mathrm{MPa})$ & $\kappa^{n p}(\mathrm{MPa})$ & $C_{10}^{\text {af }}(\mathrm{MPa})$ & $C_{20}^{\text {af }}(\mathrm{MPa})$ & $\kappa^{\text {af }}(\mathrm{MPa})$ \\
\hline 0.0033 & 67.16 & 0.038 & 0.058 \\
\hline
\end{tabular}

Note Parameters have been extracted by fitting tensile experimental curves taken from the literature. The ground matrix of the $\mathrm{np}$ was characterized by data reported for shear and compression tests of NP tissue, ${ }^{9,29,30}$ and the ground matrix of the AF by fitting the data for radial tensile test of the AF tissue. ${ }^{16}$ 

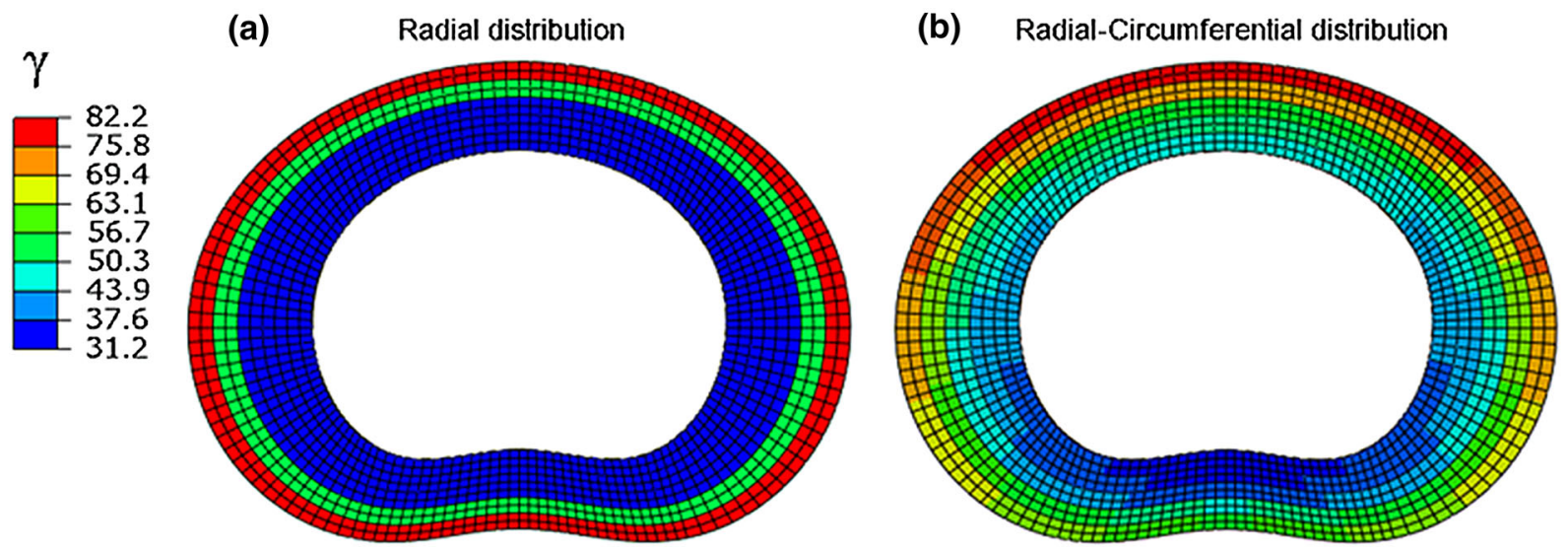

FIGURE 3. Distribution of $\gamma$ over the cross-section of the disk for the model with a radial distribution (RDM, a) and a radialcircumferential distribution of the lamellae stiffness (RCDM, b).

oped for this purpose. ${ }^{34} \mathrm{~A}$ similar approach was also used to determine e.g., the residual strain in the cornea, based on images of the eye in its stressed state. ${ }^{45}$ Its aim is to compute the residual strain configuration in the annulus by an iterative approach. Each iteration is composed of a simulation, where the external load is the internal swelling pressure. By a decomposition of the motion, the method computes the residual annulus deformation gradient, which equilibrates the internal swelling pressure and results in the defined disk geometry (as would be obtained from e.g., MR imaging). The swelling pressure was assumed $0.1 \mathrm{MPa}^{50}$ (internal pressure exhibited in vivo in supine posture). After this reference state was computed, four loading cases were applied on the pre-stressed model: Axial elongation/ compression $(1 \mathrm{~mm})$, flexion/extension $\left(5^{\circ}\right)$, left/right lateral bending $\left(3^{\circ}\right)$ and left/right axial torsion $\left(2.5^{\circ}\right) .{ }^{20}$ The stress distribution due to NP swelling was compared by analysis of the maximum and minimum principal stresses and pressure $\left(p=-\left(\sigma_{1}+\sigma_{2}+\sigma_{3}\right) / 3\right.$, where $\sigma_{i}$ are the principal stresses). Furthermore, the fiber tensioning was investigated in the loaded scenario by looking at the maximum principal stress distribution in the AF. In addition, the total proportion of the annulus volume with fiber tensioning was calculated as the annulus volume ration (avr). For the analysis of the results, unless otherwise specified, the RCDM was taken as the reference model and the relative change is referred to the value at the maximum displacement/ rotation simulated.

\section{RESULTS}

\section{Reference State}

The stress distribution due to the application of an initial swelling pressure was different for each model (Fig. 4). The maximum principal stresses for the
HDM-AV were concentrated on the inner part of the $\mathrm{AF}$, whereas they were on the outer annulus for the RDM, and in particular at the posterior recess. The RCDM showed a more homogeneous stress distribution compared to the HDM and the RDM, especially in the lateral regions of the annulus. The maximum principal stress was lower for the RCDM compared to the RDM $(-170 \%)$ but larger than for the HDM-AV model $(+20.5 \%)$. The minimum principal stress had similar trends for the three models, with larger absolute values for the RDM compared to the RCDM and HDM. The pressure had similar pattern, with a larger absolute value for the RDM $(+123.3 \%)$ and a smaller for the HDM-AV $(-53.4 \%)$.

\section{Fibre Tensioning}

In general, the pattern of fiber tensioning was different in the three models for each loading case (Figs. 5 and 6). RDM and RCDM showed areas of fiber tensioning on the outer lamellae of the AF, whereas for the HDM-AV mostly the inner annulus was involved. However, for compression and tension, stresses were concentrated in the posterior outer lamellae of the HDM-AV. Except for compression, the RCDM had a lower maximum principal stress than the RDM (max difference in torsion $-87.3 \%$ ), but larger than the HDM-AV model (maximum difference in flexion $+204.1 \%$ ). In compression, the RCDM had lower stresses than the HDM-AV $(-43.5 \%)$ and the RDM $(-86.5 \%)$.

Except for tension, the volume of the annulus experiencing fiber tensioning was larger for the HDMAV (min avr in lateral bending 0.86, max avr in left torsion 0.97) compared to the RDM (min avr in right torsion 0.42, max avr in extension 0.74) and RCDM (min avr in right torsion 0.59, max avr in compression $0.86)$. 
HDM-AV

Max principal stress

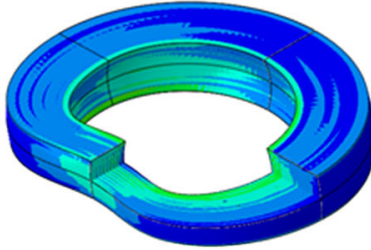

$\max$ stress $=0.66 \mathrm{MPa}$ $\min$ stress $=-0.02 \mathrm{MPa}$

Min principal stress

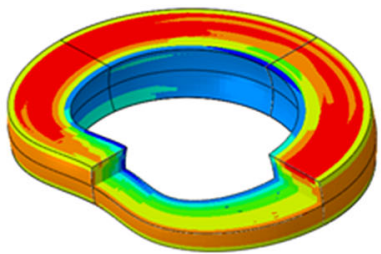

max stress $=0.03 \mathrm{MPa}$ $\min$ stress $=-0.11 \mathrm{MPa}$

Pressure

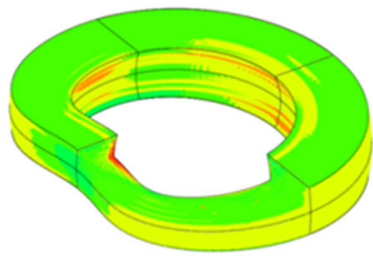

$\max$ stress $=0.04 \mathrm{MPa}$ $\min$ stress $=-0.09 \mathrm{MPa}$

$\min$
RDM

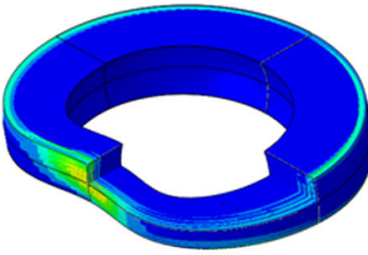

max stress $=2.24 \mathrm{MPa}$

min stress $=-0.06 \mathrm{MPa}$

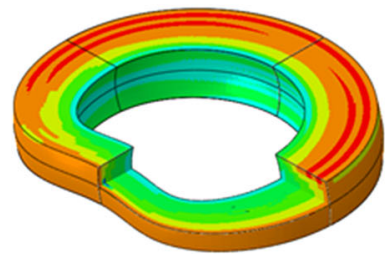

$\max$ stress $=0.03 \mathrm{Mpa}$ $\min$ stress $=-0.17 \mathrm{MPa}$

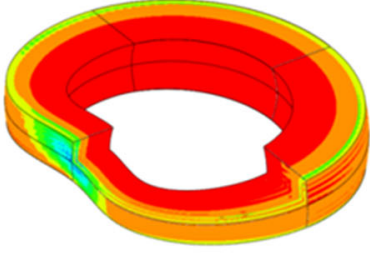

max stress $=0.08 \mathrm{MPa}$ $\min$ stress $=-0.33 \mathrm{MPa}$
RCDM
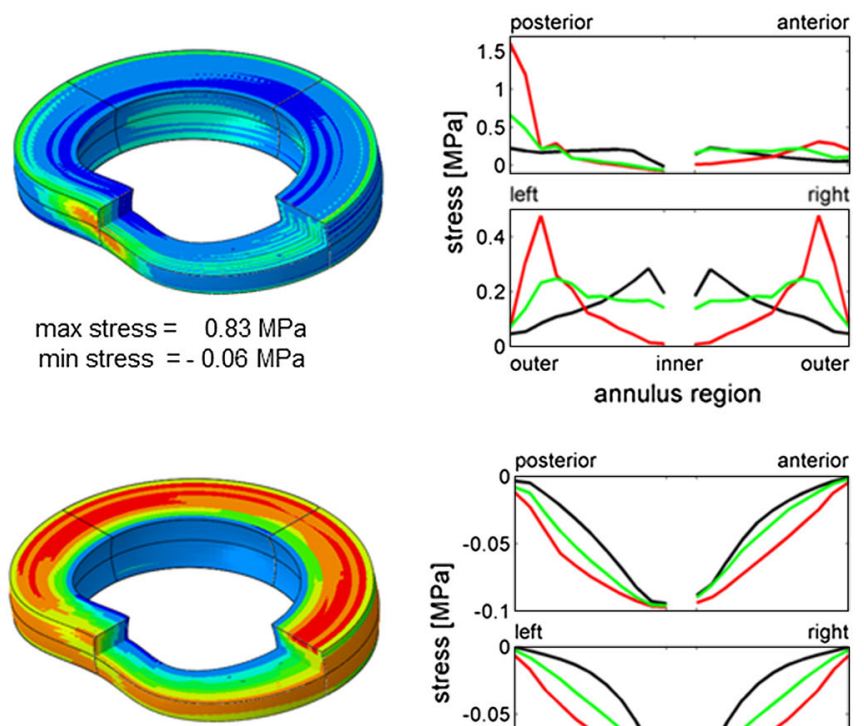

max stress $=0.03 \mathrm{MPa}$ $\min$ stress $=-0.11 \mathrm{MPa}$
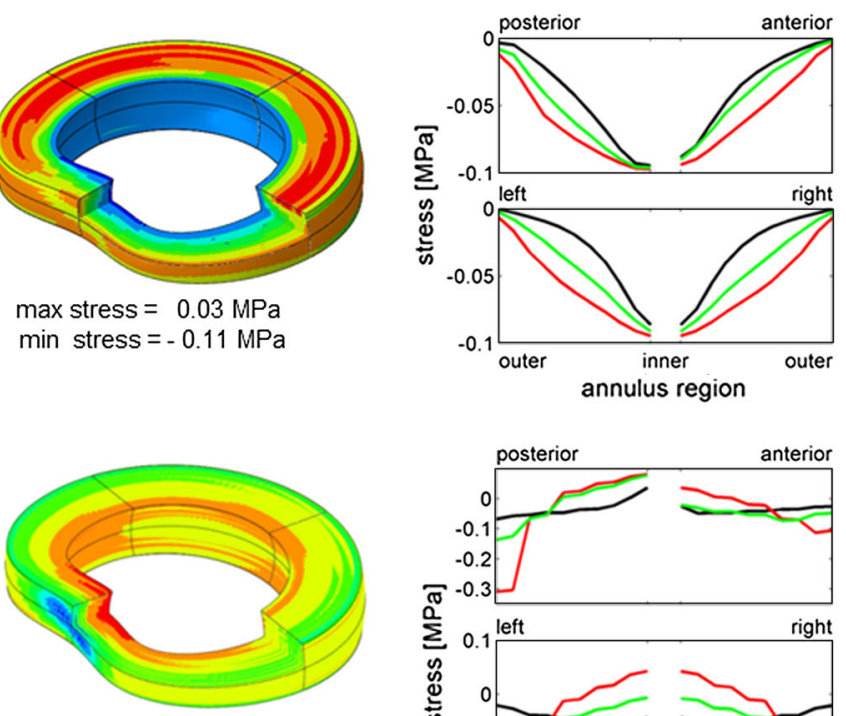

max stress $=0.08 \mathrm{MPa}$ min stress $=-0.15 \mathrm{MPa}$

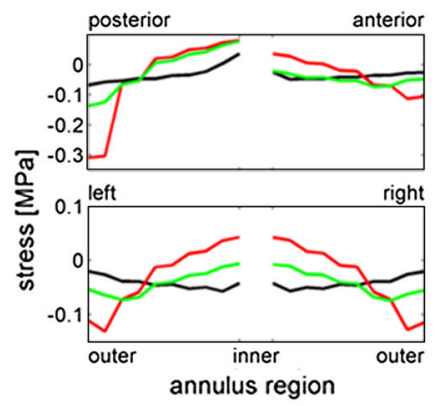

FIGURE 4. Distribution of the von Mises stresses (top) and pressure (bottom) for the model with a uniform lamellae stiffness distribution (HDM-AV, left), a radial stiffness distribution (RDM, center) and a radial-circumferential distribution (RCDM, right) at $0.1 \mathrm{MPa}$ swelling pressure. The radial distribution of the stresses at four orthogonal sections of the annulus mid-section (posterior, anterior, left and right) was compared for HDM-AV (black), RDM (red) and RCDM (green).

\section{Organ Response}

The load-displacement curves predicted by the HDM-AV (black), RDM (red) and the RCDM (green) are compared in Fig. 7. The variance of the lamellae response shown in Fig. $1 \mathrm{~b}$ is reflected in the range of variance for the organ-scale response of the HDM (gray area on Fig. 7), bounded by the response of the homogeneous models HDM-O and HDM-I. In general, the organ response was stiffer for the RDM, compared to the RCDM and the HDM-AV. Furthermore, for every loading case, the HDM-AV showed a response closer to the HDM-I than to the average response of the HDM-O and the HDM-I models. In compression, the load-displacement curves for the RDM and the RCDM showed a comparable disk response $(-5.7 \%)$ and within the range of varia- tion of the HDM. In elongation, the RDM exhibited a response stiffer than the RCDM $(+45.1 \%)$, which had a comparable response to the HDM-O $(+2.6 \%)$. In flexion, the RDM had similar response to the HDM-O $(+2.63 \%)$, and results stiffer compared to the RCDM $(+85.2 \%)$. In extension, the RDM was stiffer than the RCDM $(+17.2 \%)$ and the HDM-AV $(+73.8 \%)$. In particular, the RCDM had similar response to the HDM-O $(+0.6 \%)$. In lateral bending, the RDM resulted stiffer than the HDM-O (left $+13.6 \%$, right $+16.7 \%$ ) and the RCDM (left $+25.1 \%,+27.7$ right $\%$ ). The response of the RCDM was comparable to the HDM-O (left $-9.1 \%$, right $-8.5 \%$ ). In torsion, the RDM had similar response to the HDM-O (left $-2.9 \%$, right $-5.8 \%$ ) and, was stiffer than the RCDM (left $+41.5 \%$, right $+49.5 \%$ ). 


\section{HDM-AV}

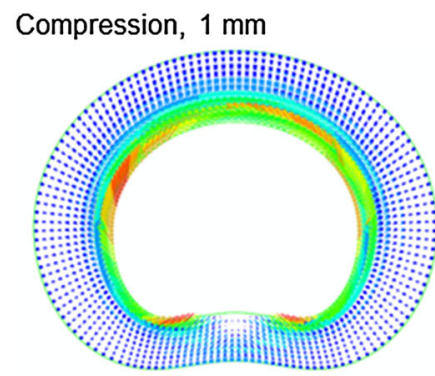

$\max$ stress $=2.77 \mathrm{MPa}$

Tension, $1 \mathrm{~mm}$

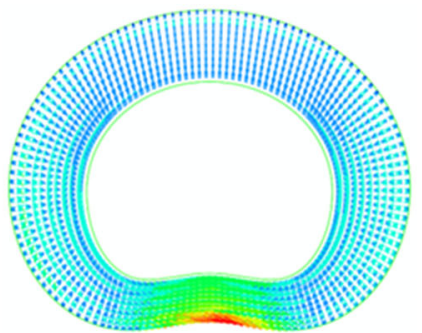

max stress $=1.97 \mathrm{MPa}$

Flexion, 5 degree

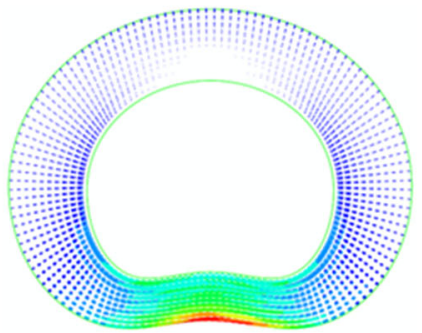

max stress $=4.94 \mathrm{MPa}$

Extension, 5 degree

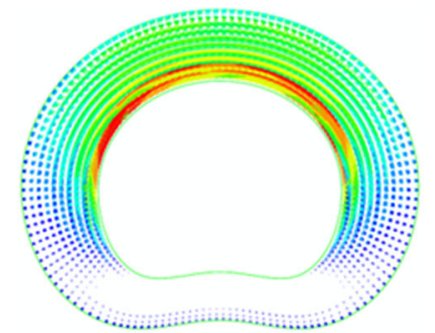

$\max$ stress $=0.69 \mathrm{MPa}$

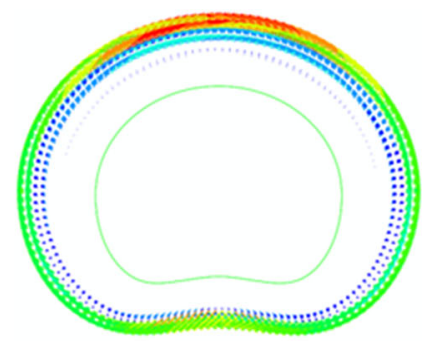

max stress $=3.60 \mathrm{MPa}$

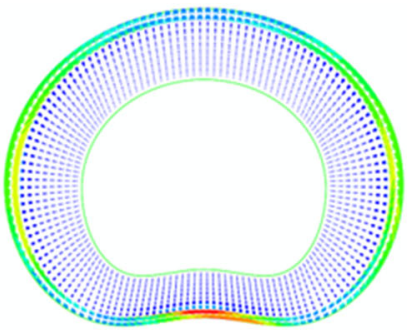

max stress $=15.63 \mathrm{MPa}$

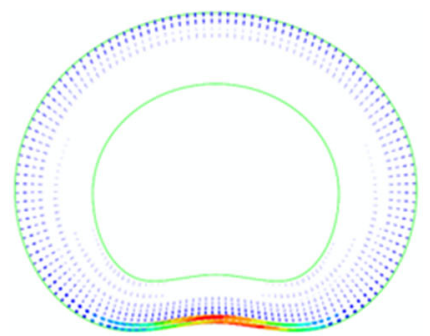

max stress $=75.13 \mathrm{MPa}$

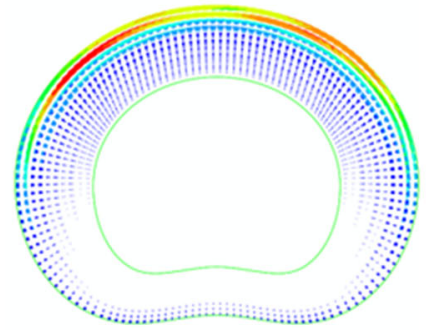

max stress $=3.38 \mathrm{MPa}$

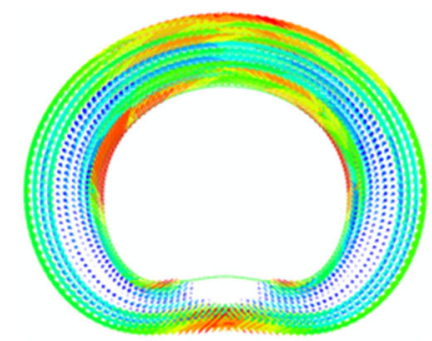

$\max$ stress $=1.93 \mathrm{MPa}$

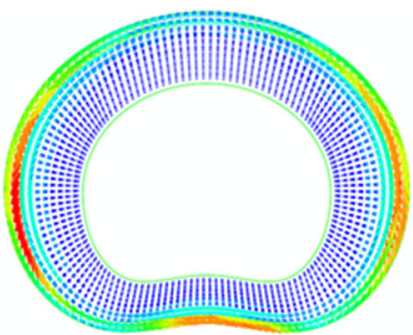

$\max$ stress $=5.48 \mathrm{MPa}$

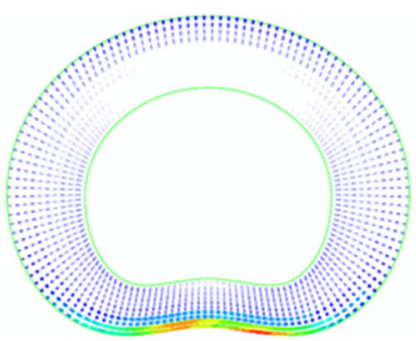

$\max$ stress $=24.68 \mathrm{MPa}$

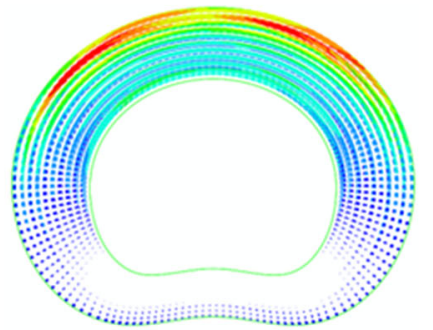

$\max$ stress $=1.68 \mathrm{MPa}$
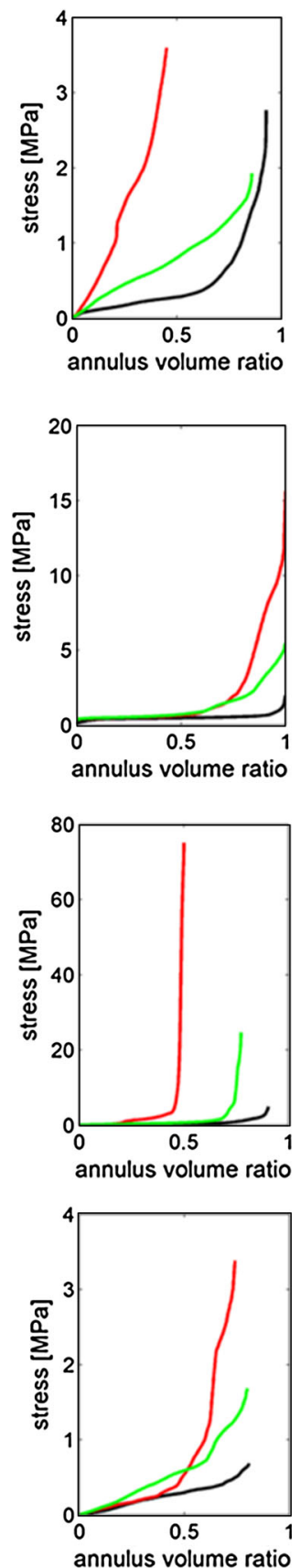

$0.0[\mathrm{MPa}]$

max stress

FIGURE 5. Vector distributions of the maximum principal stresses for models with a uniform lamellae stiffness distribution (HDMAV, left), a radial stiffness distribution (RDM, center) and a radial-circumferential distribution (RCDM, right). The minimum contour value was set to $0 \mathrm{MPa}$ to highlight the response of the fibers, which are modeled as tension-only. Far right: The distribution of stress values, normalized over the annulus volume, plotted for HDM-AV (black), RDM (red) and RCDM (green).

\section{DISCUSSION}

In this study, the influence of the spatial distribution of lamellae stiffness on the IVD response has been investigated, specifically by defining a material mapping strategy relating the local lamellae stiffness directly to the local collagen type proportion.

The presented results indicated that, depending on the modeling approach of the AF, numerical models based on lamellae mechanics predict a quite different 
HDM-AV

Lateral bending, right, 3 degree

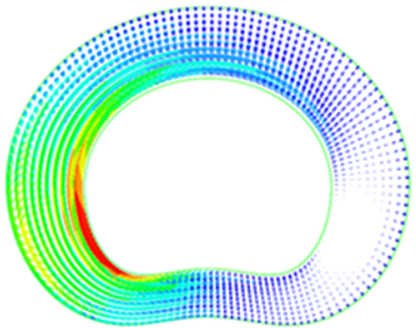

max stress $=1.02 \mathrm{MPa}$

Lateral bending, left, 3 degree

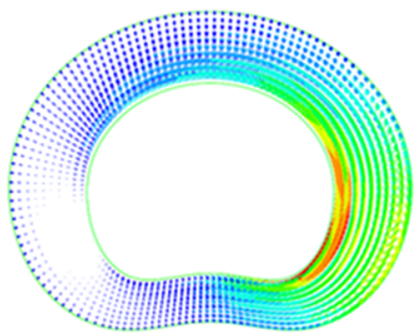

max stress $=1.02 \mathrm{MPa}$

Torsion, right, 2.5 degree

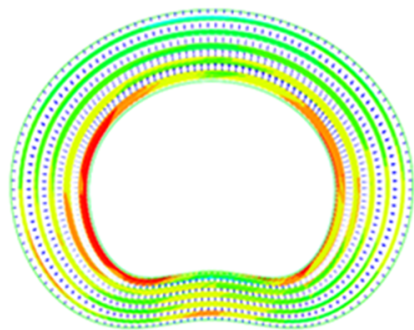

$\max$ stress $=1.02 \mathrm{MPa}$

Torsion, left, 2.5 degree

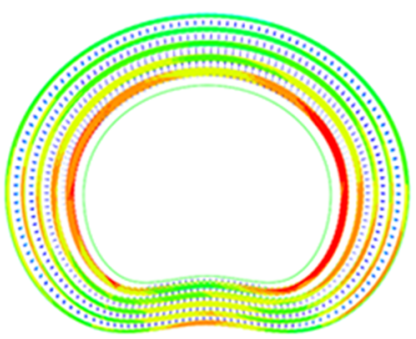

$\max$ stress $=0.78 \mathrm{MPa}$

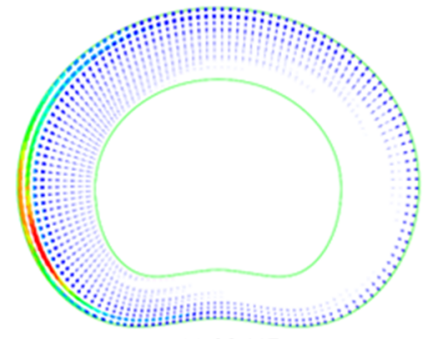

max stress $=11.06 \mathrm{MPa}$

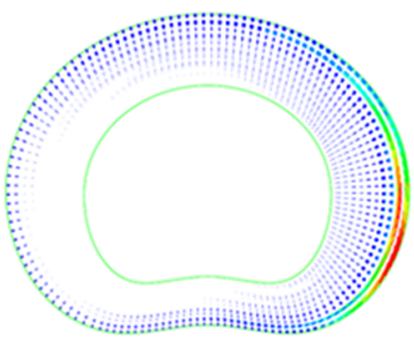

$\max$ stress $=10.40 \mathrm{MPa}$

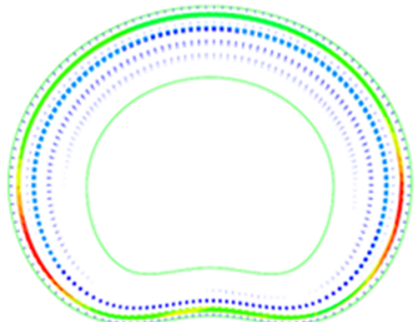

max stress $=13.19 \mathrm{MPa}$

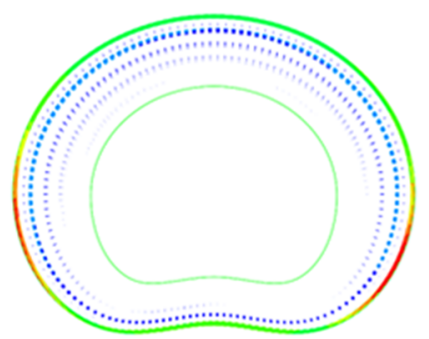

max stress $=13.32 \mathrm{MPa}$

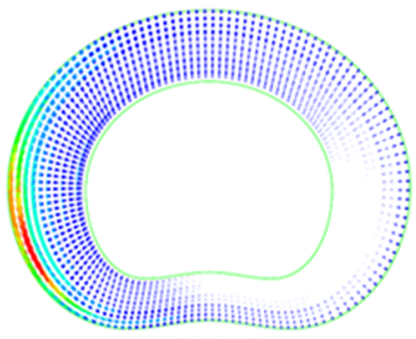

$\max$ stress $=5.69 \mathrm{MPa}$

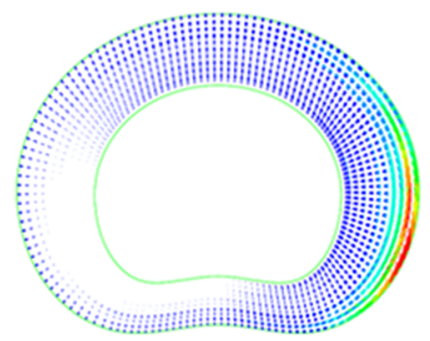

$\max$ stress $=5.87 \mathrm{MPa}$

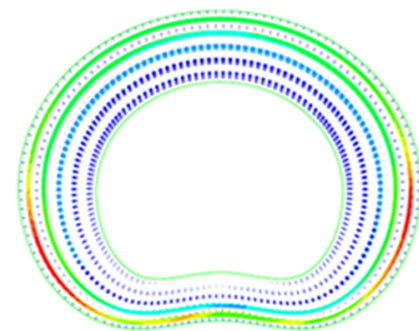

$\max$ stress $=5.92 \mathrm{MPa}$

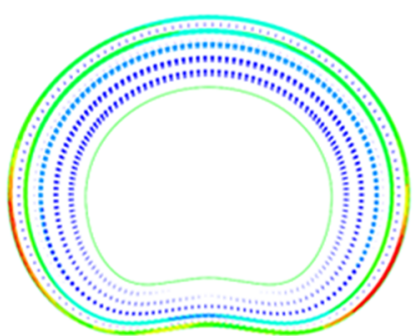

$\max$ stress $=6.30 \mathrm{MPa}$
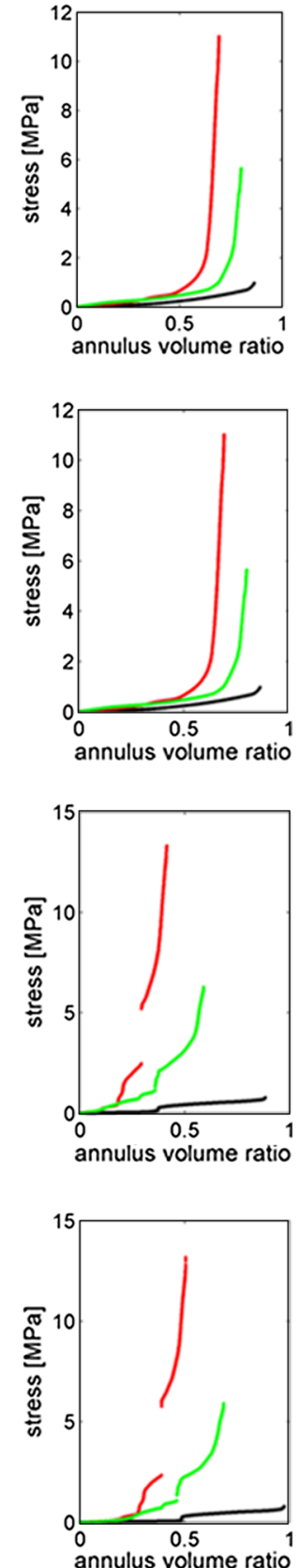

$0.0[\mathrm{MPa}]$

max stress

FIGURE 6. Vector distributions of the maximum principal stresses for models with a uniform lamellae stiffness distribution (HDM$\mathrm{AV}$, left), a radial stiffness distribution (RDM, center) and a radial-circumferential distribution (RCDM, right). The minimum contour value was set to $0 \mathrm{MPa}$ to highlight the response of the fibers, which are modeled as tension-only. Far right: The distribution of the stress values, normalized over the annulus volume, plotted for HDM-AV (black), RDM (red) and RCDM (green).

quasi-static behavior, in terms of the organ response and spatial distribution of internal variables i.e., pressure, maximum and minimum principal stress, fiber tensioning. In particular, a radial-circumferential distribution improves the load bearing capability of the system in compression by distributing the load more homogeneously on the AF lamellae. This translates to lower local maximum stresses and a different fiber contribution to the disk mechanics, compared to a system with radial distribution. Assigning the high 

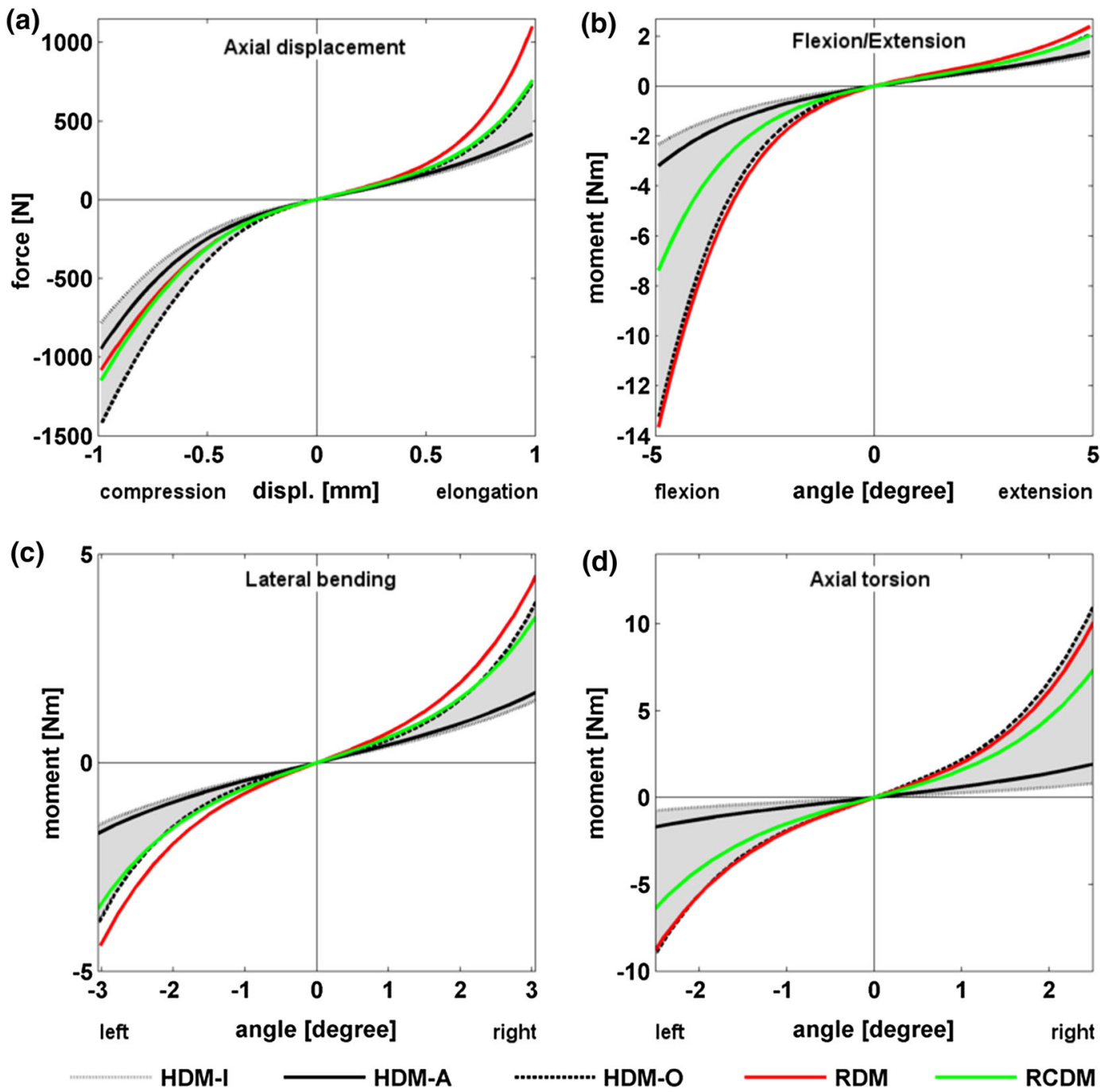

FIGURE 7. Load-Displacement curves predicted by the FE models with a homogeneous (HDMs, black line), a radial (RDM, red line) and a radial-circumferential (RCDM, green line) lamellae stiffness distribution. The area of variance (gray) for the model with a homogeneous lamellae stiffness is bounded by the HDM with lamellae response equivalent to the posterior internal lamellae of the RDM (HDM-I, dotted line), and the HDM with lamellae response equivalent to the anterior external lamellae of the RDM (HDM-O, dashed line). See also Fig. 2.

stiffness of the anterior external lamellae homogenously to the whole AF (Fig. 1a) did not produce the expected limit on the organ-scale response for the system i.e., tension, extension, lateral bending (HDMO). This is likely due to the initial stress distribution following the pre-stress analysis step. Areas of stress concentration following pre-stress were predicted mostly in the lamellae surrounding the nucleus in the HDMs, whereas in the RDM the outer lamellae were the main area of stress concentration. This translates to a larger moment arm for the (integrated) area of stress in the RDM than in the HDMs models, which together with the larger initial stresses $(+53 \%)$ entails a stiffer organ response for the RDM than for the HDM-O. However, the stress distribution induced by the swelling pressure seemed to be less relevant for compression, where the HDM-O resulted in a stiffer organ response than the RDM and RCDM. Furthermore, although a similar axial response was predicted by the RDM and the RCDM, the maximum principal stresses were smaller for the RCDM than for the RDM. Studies ${ }^{43}$ reported values of failure stresses for the anterior lamellae of $10.3 \pm 8.4 \mathrm{MPa}$ which is five times higher than the maximum principal stress at $1 \mathrm{~mm}$ axial compression from the RCDM. Compression is considered to be the main loading condition experienced by the disk during daily activities ${ }^{49}$ and our analysis supports that the compositional/structural organization of the lamellae is optimized to reduce the local stress concentration in compression. However, the maximum principal stresses for the RCDM in flexion were more than two times the failure stresses 
for the anterior lamellae. This is likely due to the absence of structures such as anterior and posterior longitudinal ligaments in the model, which would contribute to resisting the flexion/extension bending moment. ${ }^{24,41}$

The adaptation and arrangement of basic biological components in order to optimally distribute the stresses in load bearing structures is implied by Wolff's law. The relationship between structural organization and the applied loads has been demonstrated to be valid in several biological systems e.g., bone and blood vessels. ${ }^{25}$ Although not the principal goal of our study, the results imply that also the IVD experiences a similar adaptation, confirming previous study from Noailly et al., ${ }^{38}$ who explored different assumptions related to adaptation of the collagen fiber angles in adjacent lamellae. The IVD, as any biological structure, undergoes changes dictated by both the load history and the physiological aging of the body., ${ }^{4,11,37,46}$ Antoniou et al. ${ }^{6}$ studied the changes in the biosynthesis and denaturation of the extracellular matrix and identified three major matrix turnover phases: Growth ( $0-15$ years), maturation and aging (15-40 years), and degeneration and fibrotic (40-80 years), similar to the age groups explored in Brickley-Parson and Glimcher. ${ }^{7}$ In particular, after growth the vascular supply through the vertebral margins strongly diminishes and nutrients are delivered by diffusive processes from the free margins of the annulus, still vascularized, and by the diurnal fluid flow through the endplates. ${ }^{15,22}$ During maturation and aging, the disk would be similar to a closed system, where processes of remodeling and healing are limited to the boundaries. Therefore, the different response of the anterior and posterior region of the AF (Fig. 1) could be due to adaptation during the maturation and aging phase.

The changes of lamellae stiffness were related only to $\left[\mathrm{Coll}_{\mathrm{I}}\right]$, since bundles of $\mathrm{Coll}_{\mathrm{I}}$ fibers are mostly present in tissues having a direct load bearing function. We assumed therefore they have a similar function in the lamellae and their change in content directly influences the lamellae stiffness. However, while in maturation, an increase of $\mathrm{Coll}_{\mathrm{I}}$ in the anterior-inner region is reflected by an increase of the lamellae stiffness, the opposite occurs in the posterior-outer region of the AF (Figs. 1a and 2a). This phenomenon could be due to the fact that, during the aging and degeneration of the disk, bundles of $\mathrm{Coll}_{\mathrm{II}}$ are replaced by

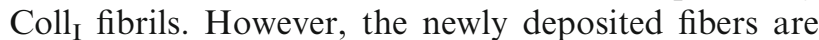
not aligned along a preferential direction, but rather they form a random fibrous network ${ }^{3,23,37}$ which results in a different contribution to the lamellae mechanics. In lamellae previously rich in Coll $\mathrm{II}_{\mathrm{II}}$, these randomly aligned Coll fibers would still enhance the mean stiffness of the tissue (i.e., intrinsic fiber prop-

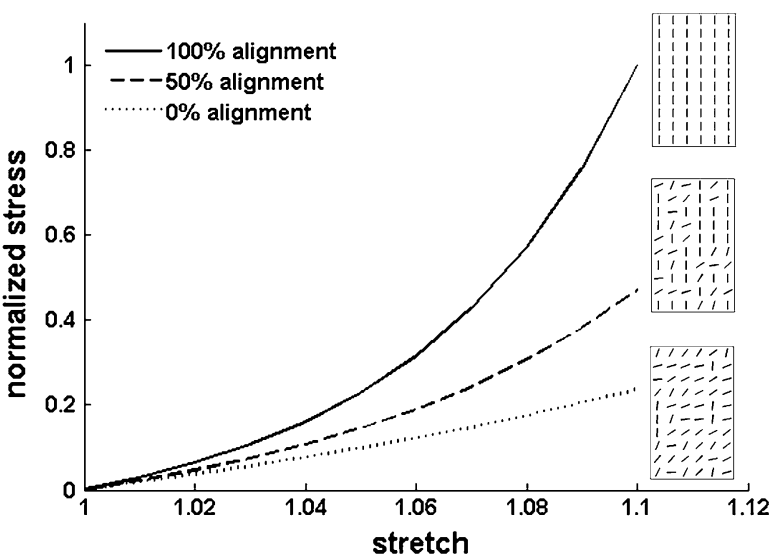

FIGURE 8. Stress-stretch curves from simulations of uniaxial tensile tests, assuming $100 \%$ fiber alignment (solid line), $50 \%$ fiber alignment (dashed line) and $0 \%$ fiber alignment (dotted line), with respect to the tensile loading direction (vertical). The example assumes the same type fibers with no reference to a specific kind of collagen. Stresses were normalized with respect to the maximum value obtained for $100 \%$ fiber alignment. Simulations were performed with $C_{10}=0.038$ (MPa), $C_{20}=0.058$ (MPa), $\kappa=9.52(\mathrm{MPa}), \mu=0.01$ (MPa) and $\gamma=32$.

erties dominate). However, if similar changes occur in lamellae with a previously high content of aligned Coll fibers, freshly deposited fibers would not increase the local lamellae stiffness (i.e., alignment effects dominate) (Fig. 8).

Although the assumption of a correlation between lamellae stiffness and local collagen type is not based on any direct experimental evidence, but rather the synthesis of observations from two studies, it has been demonstrated that the mechanical properties of the AF and NP can be generally related to the biochemical composition. ${ }^{5,21,39}$ By changing the swelling and diffusion properties of the IVD, the chemical-electrical behavior of the tissue plays also a fundamental role in the stress distribution of the loads through the system. ${ }^{27}$ In the present study, we neglected these aspects, considering variation in swelling pressure during loading transients to be secondary to direct mechanical effects. ${ }^{47}$

Although the chemical-electrical behavior also changes with age, we did not specifically study the process of disk aging and degeneration. The distribution of $\left[\mathrm{Coll}_{\mathrm{I}}\right]$ was taken from different age groups, however no conclusion can be made about the influence of age on the disk response because this would involve changes also in geometry, which were not considered in the study. Moreover, the reference stress-stretch curves were related to the mature specimen $^{26}[57.9$ (mean) \pm 15.4 (SD) years] and do not completely represent all the possible physiological variance; further detailed analyses of the constitutive determinants of AF lamella properties should be encouraged. 

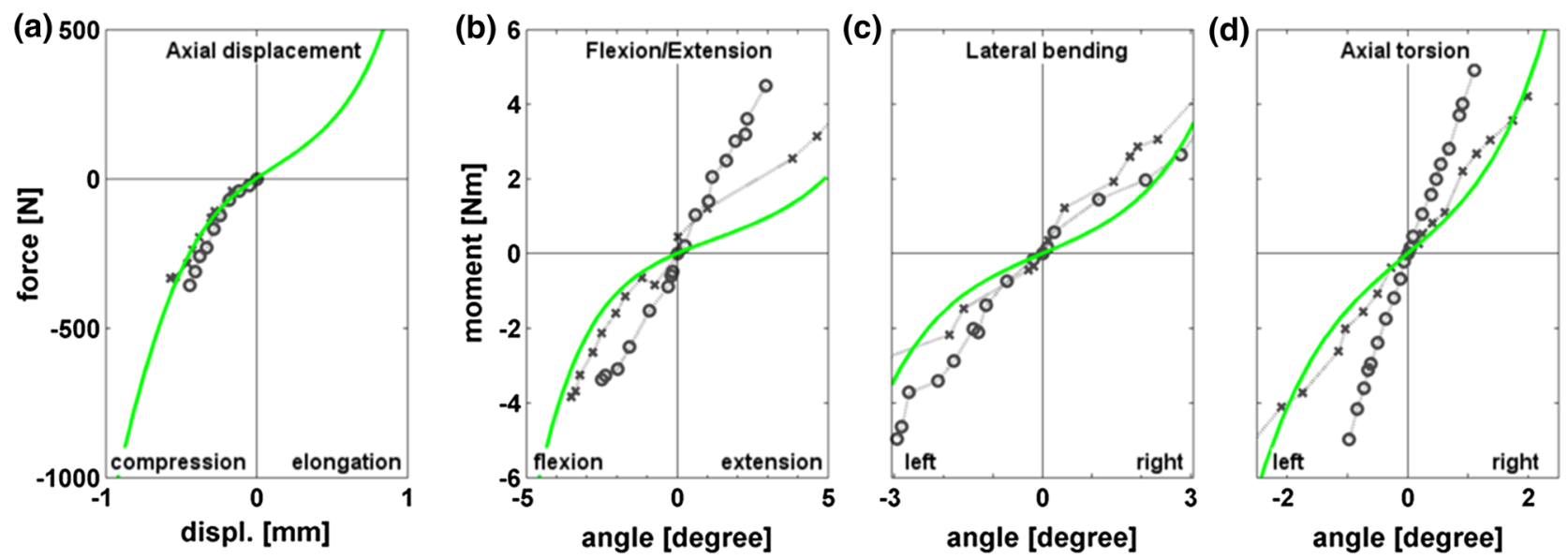

*Wang-L2L3-sample 1

- Wang-L2L3-sample 2

RCDM

FIGURE 9. Load-Displacement curves predicted by the FE model with a radial-circumferential lamellae stiffness distribution (RCDM) and analog experiments on L2-L3 disks from literature. ${ }^{48}$

To use numerical analysis to investigate the effectiveness of repair strategies or to model the disk cell response to targeted treatments, the micro-mechanical environment should be correctly described, which implies the need for an accurate description of the local tissue properties. Nonlinear models with a discretization grid resolving the single fibers would be computationally prohibitive, whereas heterogeneous phenomenological models need to be calibrated carefully, and may have too many valid parameter combinations which are then difficult to relate to specific characteristic of the tissue. ${ }^{40}$ Models describing purely the structural organization of the tissue components ${ }^{13,31}$ do not consider the change in mechanical properties due to the tissue composition, which is relevant when considering tissue aging and remodeling. The outcome of the numerical analysis suggests that investigation of the link between the collagen type and the local lamellae mechanical response, with a particular focus on the effect of age, would provide valuable information on natural disk function or even guidance for future tissue engineering applications.

In our approach, the discretization of the AF dictates the local proportion of collagen type for, and hence the local stiffness assigned to, each element. In order to verify the influence of discretization on the numerical solution, we ran a mesh sensitivity study with one 6-layer and one 16-layer RCDM. Both models had similar organ response to the 10-layer RCDM, however the 6-layer model differed for some load cases i.e., elongation ( $\max$ error $-7.5 \%$ ), flexion (max error $+12.1 \%)$, right lateral bending (max error $9.8 \%)$, left axial torsion $(+23 \%)$, thus indicating that the approach needs at least a seeding size of circa
$1 \mathrm{~mm}$, which agrees with mesh sensitivity studies for dynamic simulations. ${ }^{33}$

Although the model was not created based on a real disk geometry, we compared qualitatively the organ response with data from literature ${ }^{48}$ (Fig. 9). In general, the response of the RCDM had a good correlation with the experiment (Pearson's product-moment correlation, $R>0.79, p<0.001)$ with the exception of the model response in flexion $(R=0.75, p<0.001)$. This is most likely due to the absence of the posterior and anterior ligaments. Experimental studies have shown that the presence of these ligaments can change the mechanical response of the system. ${ }^{24}$ In our study, we neglected these structures in order to highlight the influence of collagen distribution in the annulus. The comparison of the internal stresses for $500 \mathrm{~N}$ compressive load to the stress profilometry values reported in previous studies ${ }^{36,38}$ (Fig. 10) showed a good correlation $(R>0.75, p<0.001)$, although the assumption of the swelling related only to the NP was visible in the sudden drop of the stresses beyond the NP boundary.

In conclusion, the influence of a heterogeneous AF lamella stiffness on the organ-scale mechanical response of numerical models of the IVD has been investigated. Lamellar properties were assigned based a mapping strategy relating stiffness to local collagen type. The results indicate that the local stiffness variation in AF tissue has an important influence on disk mechanics, therefore an assumption of homogeneous lamellae stiffness could be erroneous. The study has also indirectly highlighted that the structural organization of the mature IVD could be a consequence of adaptation to the stresses induced by the applied loads. 

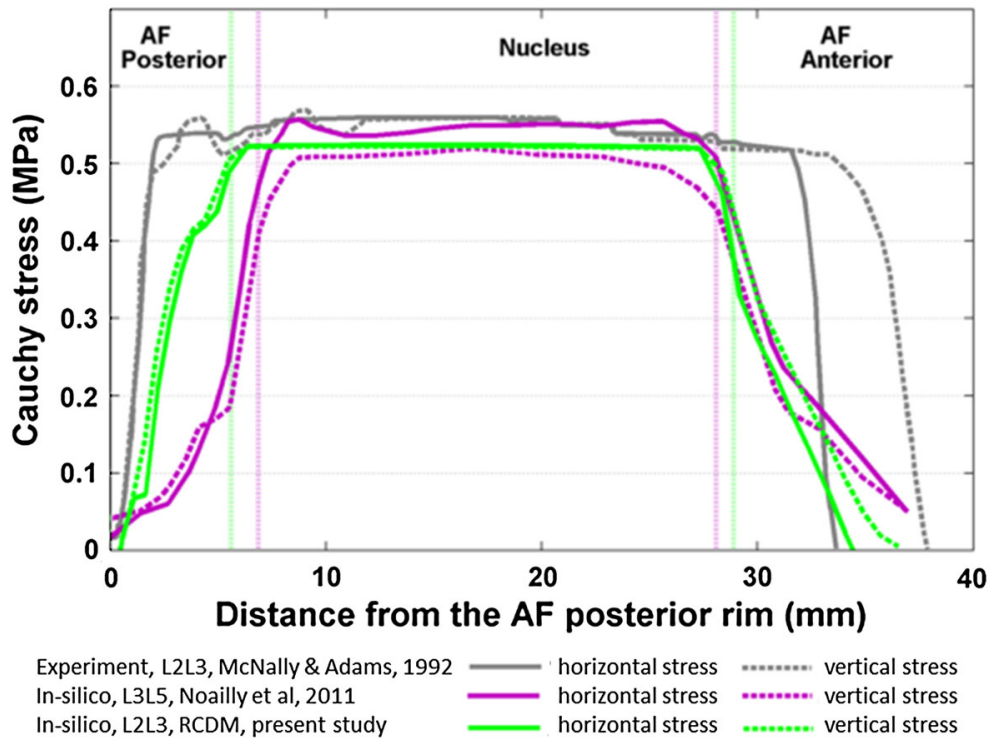

FIGURE 10. Stress profilometry vs. distance from the AF posterior rim for $500 \mathrm{~N}$ axial compression. Vertical and horizontal stresses of the RCDM model (green) were compared to the experiment of McNally and Adams ${ }^{36}$ (gray) and the numerical model of Noailly et $a^{38} .^{38}$ (magenta). Vertical dotted lines represent the NP boundaries for the two numerical models.

\section{ACKNOWLEDGMENT}

Funding for this research project was provided by the European Union through a Marie Curie action (FPT7-PITN-GA-2009-238690-SPINEFX).

\section{CONFLICT OF INTEREST}

The authors have no conflict of interest to declare.

\section{REFERENCES}

${ }^{1}$ Acaroglu, E. R., J. C. Iatridis, L. A. Setton, R. J. Foster, V. C. Mow, and M. Weidenbaum. Degeneration and aging affect the tensile behavior of human lumbar anulus fibrosus. Spine 20:2690-2701, 1995.

${ }^{2}$ Adams, M. A., and T. P. Green. Tensile properties of the annulus fibrosus. I. The contribution of fibre-matrix interactions to tensile stiffness and strength. Eur. Spine J. 2:203-208, 1993.

${ }^{3}$ Adams, M. A., and P. J. Roughley. What is intervertebral disc degeneration, and what causes it? Spine 31:2151-2161, 2006.

${ }^{4}$ Adams, P., D. R. Eyre, and H. Muir. Biochemical aspects of development and ageing of human lumbar intervertebral discs. Rheumatol. Rehabil. 16:22-29, 1977.

${ }^{5}$ Aladin, D. M., K. M. Cheung, A. H. Ngan, D. Chan, V. Y. Leung, C. T. Lim, K. D. K. Luk, and W. W. Lu. Nanostructure of collagen fibrils in human nucleus pulposus and its correlation with macroscale tissue mechanics. J. Orthop. Res. 28:497-502, 2010.

${ }^{6}$ Antoniou, J., T. Steffen, F. Nelson, N. Winterbottom, A. P. Hollander, R. A. Poole, M. Aebi, and M. Alini. The human lumbar intervertebral disc. Evidence for changes in the biosynthesis and denaturation of the extracellular matrix with growth, maturation, ageing, and degeneration. J. Clin. Invest. 98:996-1003, 1996.

${ }^{7}$ Brickley-Parsons, D., and M. J. Glimcher. Is the chemistry of collagen in intervertebral discs an expression of Wolff's Law? A study of the human lumbar spine. Spine 9:148-163, 1984.

${ }^{8}$ Cassidy, J. J., A. Hiltner, and E. Baer. Hierarchical structure of the intervertebral disc. Connect. Tissue Res. 23:75-88, 1989.

${ }^{9}$ Cloyd, J. M., N. R. Malhotra, L. Weng, W. Chen, R. L. Mauck, and D. M. Elliott. Material properties in unconfined compression of human nucleus pulposus, injectable hyaluronic acid-based hydrogels and tissue engineering scaffolds. Eur. Spine J. 16:1892-1898, 2007.

${ }^{10}$ Coventry, M. B., and R. K. Ghormley. The intervertebral disc: its microscopic anatomy and pathology. Part I. Anatomy, Development, and Physiology. J. Bone Joint Surg. 27:105-112, 1945.

${ }^{11}$ Coventry, M. B., R. K. Ghormley, and J. W. Kernohan. The intervertebral disc: its microscopic anatomy and pathology. Part II. Changes in the intervertebral disc concomitant with age. J. Bone Joint Surg. 27:233-247, 1945.

${ }^{12}$ Ebara, S., J. C. Iatridis, L. A. Setton, R. J. Foster, V. C. Mow, and M. Weidenbaum. Tensile properties of nondegenerate human lumbar anulus fibrosus. Spine 21:452-461, 1996.

${ }^{13}$ Elliott, D. M., and L. A. Setton. A linear material model for fiber-induced anisotropy of the anulus fibrosus. $J$. Biomech. Eng. 122:173-179, 2000.

${ }^{14}$ Eyre, D. R., and H. Muir. Quantitative analysis of types I and II collagens in human intervertebral discs at various ages. Biochim. Biophys. Acta 492:29-42, 1977.

${ }^{15}$ Ferguson, S. J., K. Ito, and L.-P. Nolte. Fluid flow and convective transport of solutes within the intervertebral disc. J. Biomech. 37:213-221, 2004.

${ }^{16}$ Fujita, Y., N. A. Duncan, and J. C. Lotz. Radial tensile properties of the lumbar annulus fibrosus are site and degeneration dependent. J. Orthop. Res. 15:814-819, 1997. 
${ }^{17}$ Galante, J. O. Tensile properties of the human anulus fibrosus. Acta Orthop. Scand. 100[Suppl]:4-91, 1967.

${ }^{18}$ Gelse, K., E. Pöschl, and T. Aigner. Collagens-structure, function, and biosynthesis. Adv. Drug Deliv. Rev. 55:15311546, 2003.

${ }^{19}$ Gu, W. Y., X. G. Mao, R. J. Foster, M. Weidenbaum, V. C. Mow, and B. A. Rawlins. The anysotropic hydraulic permeability of human lumbar anulus fibrosus. Influence of age, degeneration, direction, and water content. Spine 24:2449-2455, 1999.

${ }^{20}$ Guoan, L., S. Wang, P. Passias, Q. Xia, G. Li, and K. Wood. Segmental in vivo vertebral motion during functional human lumbar spine activities. Eur. Spine J. 18:1013-1021, 2009.

${ }^{21}$ Han, W. M., N. L. Nerurkar, L. J. Smith, N. T. Jacobs, R. L. Mauck, and D. M. Elliott. Multi-scale structural and tensile mechanical response of annulus fibrosus to osmotic loading. Ann. Biomed. Eng. 40:1610-1621, 2012.

${ }^{22}$ Hassler, O. The human intervertebral disc. A micro-angiographical study on its vascular supply at various age. Acta Orthop. Scand. 40:765-772, 1970.

${ }^{23}$ Herbert, C. M., K. A. Lindberg, M. I. Jayson, and A. J. Bailey. Changes in the collagen of human intervertebral discs during ageing and degenerative disc disease. J. Mol. Med. 1:79-91, 1975.

${ }^{24}$ Heuer, F., H. Schmidt, Z. Klezl, L. Claes, and H.-J. Wilke. Stepwise reduction of functional spinal structures increase range of motion and change lordosis angle. J. Biomech. 40:271-280, 2007.

${ }^{25}$ Holzapfel, G. A., and R. W. Ogden. Mechanics of Biological Tissues. Wien: Springer, 2006, 522 pp.

${ }^{26}$ Holzapfel, G. A., C. A. Schulze-Bauer, G. Feigl, and P. Regitnig. Single lamellar mechanics of the human lumbar anulus fibrosus. Biomech. Model. Mech. 3:125-140, 2005.

${ }^{27}$ Iatridis, J. C., J. L. Laible, and M. H. Krag. Influence of fixed charge density magnitude and distribution on the intervertebral disc: applications of a poroelastic and chemical electric (PEACE) model. J. Biomech. Eng. 125:1224, 2003.

${ }^{28}$ Iatridis, J. C., J. J. MacLean, M. O’Brien, and I. A. Stokes. Measurements of proteoglycans and water content distribution in human lumbar intervertebral discs. Spine 32:1493-1497, 2007.

${ }^{29}$ Iatridis, J. C., M. Weidenbaum, L. A. Setton, and V. C. Mow. Is the nucleus pulposus a solid or a fluid? Mechanical behaviors of the nucleus pulposus of the human intervertebral disc. Spine 21:1174-1184, 1996.

${ }^{30}$ Johannessen, W., and D. M. Elliott. Effects of degeneration on the biphasic material properties of human nucleus pulposus in confined compression. Spine 30:E724-E729, 2005.

${ }^{31}$ Malandrino, A., J. Noailly, and D. Lacroix. Regional annulus fibre orientations used as a tool for the calibration of lumbar intervertebral disc finite element models. Comput. Methods Biomech. Biomed. Eng. 16:923-928, 2012.

${ }^{32}$ Marchand, F., and A. M. Ahmed. Investigation of the laminate structure of the lumbar disc anulus fibrosus. Spine 15:402-410, 1990.

${ }^{33}$ Marini, G., and S. J. Ferguson. Nonlinear numerical analysis of the structural response of the intervertebral disc to impact loading. Comput. Methods Biomech. Online, 2012.
${ }^{34}$ Marini, G., H. Studer, and S. J. Ferguson. Influence of the swelling pressure in a bottom-up description of the intervertebral disc. In: 8th European Solid Mechanics Conference, Graz, Austria, 2012.

${ }^{35}$ Marini, G., I. Koh, H. Studer, and S. J. Ferguson. An algorithm for modeling the fibre distribution of the annulus fibrosus within arbitrarily-aligned meshes. In: 10th International Symposium on Computer Methods in Biomechanics, Berlin, Germany, 2012.

${ }^{36}$ McNally, D. S., and M. A. Adams. Internal intervertebral disc mechanics as revealed by stress profilometry. Spine 17:66-73, 1992.

${ }^{37}$ Naylor, A., F. Happey, and T. Macrae. The collageneous changes in the intervertebral disk with age and their effect on its elasticity. Brit. Med. J. 2(4887):570-574, 1954.

${ }^{38}$ Noailly, J., J. A. Planell, and D. Lacroix. On the collagen criss-cross angles in the annuli fibrosis of lumbar spine finite element models. Biomech. Model. Mech. 10:203-219, 2011.

${ }^{39}$ Perie, D. S., J. J. Maclean, J. P. Owen, and J. C. Iatridis. Correlating material properties with tissue composition in enzymatically digested bovine annulus fibrosus and nucleus pulposus tissue. Ann. Biomed. Eng. 34:769-777, 2006.

${ }^{40}$ Schmidt, H., F. Heuer, U. Simon, A. Kettler, A. Rohlmann, L. Claes, and H.-J. Wilke. Application of a new calibration method for a three-dimensional finite element model of a human lumbar annulus fibrosus. Clin. Biomech. 21:337-344, 2006.

${ }^{41}$ Sharma, M., N. A. Langrana, and J. Rodriguez. Role of ligaments and facets in lumbar spinal stability. Spine 20:887-900, 1995.

${ }^{42}$ Shirazi-Adl, A., A. M. Ahmed, and S. C. Shrivastava. Mechanical response of a lumbar motion segment in axial torque alone and combined with compression. Spine 11:914-927, 1986.

${ }^{43}$ Skaggs, D. L., M. Weidenbaum, J. C. Iatridis, A. Ratcliffe, and V. C. Mow. Regional variation in tensile properties and biochemical composition of the human lumbar anulus fibrosus. Spine 19:1310-1319, 1994.

${ }^{44}$ Skrzypiec, D., M. Tarala, P. Pollintine, P. Dolan, and M. A. Adams. When are intervertebral discs stronger than their adjacent vertebrae? Spine 32:2455-2461, 2007.

${ }^{45}$ Studer, H. P., H. Riedwyl, C. A. Amstutz, J. V. M. Hanson, and P. Büchler. Patient-specific finite-element simulation of the human cornea: a clinical validation study on cataract surgery. J. Biomech. 46:751-758, 2013.

${ }^{46}$ Twomey, L., and J. Taylor. Age changes in lumbar intervertebral discs. Acta Orthop. Scand. 56:496-499, 1985.

${ }^{47}$ Urban, J. P. G., and A. Maroudas. Swelling of the intervertebral disc in vitro. Connect. Tissue Res. 9:1-10, 1981.

${ }^{48}$ Wang, S. P., H. R. Gadikota, J. Miao, Y. H. Kim, K. B. Wood, and G. Li. A combined numerical and experimental technique for estimation of the forces and moments in the lumbar interverterbal disc. Comput. Method. Biomech. Online, 2012.

${ }^{49}$ White, A. A., and M. M. Panjabi. Clinical Biomechanics of the Spine. Philadelphia: Lippincott Williams \& Wilkins, 1978, $752 \mathrm{pp}$

${ }^{50}$ Wilke, H.-J., P. Neef, M. Caimi, T. Hoogland, and L. E. Claes. New in vivo measurements of pressures in the intervertebral disc in daily life. Spine 24:755-762, 1999. 